\title{
Methylphenidate Effects on Cortical Thickness in Children and Adults with Attention-Deficit/Hyperactivity Disorder: A Randomized Clinical Trial
}

\author{
(D) K.B. Walhovd, (DI. Amlien, (D) A. Schrantee, (DD.A. Rohani, (DI. Groote, (D)A. Bjørnerud, (D)A.M. Fjell, and (D) Reneman
}

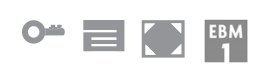

\begin{abstract}
BACKGROUND AND PURPOSE: Although methylphenidate is frequently used to treat children with attention-deficit/hyperactivity disorder, it is currently unknown how methylphenidate affects brain development. In a randomized controlled trial, we investigated whether the cortical effects of methylphenidate are modulated by age.
\end{abstract}

\begin{abstract}
MATERIALS AND METHODS: Between June 1, 2011, and June 15, 2015, we conducted a randomized, double-blind, placebo-controlled trial (Effects of Psychotropic Drugs on Developing Brain-Methylphenidate) in 99 males with attention-deficit/hyperactivity disorder (according to Diagnostic and Statistical Manual of Mental Disorders, 4th Edition, criteria) from referral centers in the greater Amsterdam area in the Netherlands. The trial was registered on March 24, 2011 (identifier NL34509.000.10) and subsequently at the Netherlands National Trial Register (identifier NTR3103). Participants (first enrolled October 13, 2011) were 10-12 years or 23-40 years of age and randomized to treatment with either methylphenidate or a placebo for 16 weeks. Our main outcome was a change in cortical thickness in predefined ROls as measured by MR imaging pre- and posttreatment.
\end{abstract}

RESULTS: We observed a time $\times$ medication $\times$ age interaction $(F[1,88.825]=4.316, P<.05)$ for the right medial cortex ROI, where methylphenidate treatment yielded less cortical thinning in children, but not in adults or the placebo groups.

CONCLUSIONS: Our finding that the effects of methylphenidate on right medial cortical thickness differ between children and adults infers that the drug affects gray matter development in this brain region. This warrants replication in larger groups with longer follow-up to determine whether this effect can also be observed in other cortical brain regions and whether it may have long-term consequences.

ABBREVIATIONS: $\mathrm{ADHD}=$ attention-deficit/hyperactivity disorder; $\mathrm{MNI}=$ Montreal Neurological Institute; RCT = randomized controlled trial

$\mathrm{T}$ he psychostimulant methylphenidate is among the drugs most frequently prescribed to children for treatment of attention-deficit/hyperactivity disorder (ADHD) ${ }^{1,2}$ Randomized controlled trials (RCTs) have shown that methylphenidate suppresses children's physical growth during treatment, ${ }^{3,4}$ but they have not included measures of brain development. However, longitudinal brain maturation studies using MR imaging indicate that children with $\mathrm{ADHD}$, on average, deviate from controls in

Received February 1, 2020; accepted after revision April 8.

From the Department of Psychology (K.B.W., I.A., D.A.R., A.B., A.M.F.), Center for Lifespan Changes in Brain and Cognition, and Institute of Physics (A.B.), Faculty of Mathematics and Natural Sciences, University of Oslo, Oslo, Norway; Departments of Radiology and Nuclear Medicine (K.B.W., A.M.F.) and Diagnostic Physics (I.G., A.B.), Oslo University Hospital, Oslo, Norway; and Department of Radiology and Nuclear Medicine (A.S., A.B., L.R.), Amsterdam UMC, Academic Medical Center, Amsterdam, the Netherlands.

This study was funded by faculty resources of the Academic Medical Center, University of Amsterdam, and grant 11.32050.26 from the European Research Area Network Priority Medicines for Children (Sixth Framework Programme).

Clinical Trial registry name and registration number: Central Committee on Research Involving Human Subjects (an independent registry, identifier NL34509.000.10) and the Netherlands National Trial Register (identifier NTR3103). their brain developmental trajectories, including regionally thinner cortices and lower basal ganglia volumes. ${ }^{5,6}$ Recent highly powered cross-sectional studies on subcortical volumes and cortical thickness and surface area have shown ADHD case-control differences, with higher effect sizes in children than adults. ${ }^{7,8}$ In these observational cross-sectional studies, the authors found no influence of psychostimulant medication on subcortical volumes or cortical thickness of the areas showing group differences, but in smaller surface areas of regions of the frontal cortex in children taking the medication. ${ }^{7,8}$ Prospective studies on children with ADHD using-versus-not using psychostimulants have yielded

Please address correspondence to Liesbeth Reneman, MD, PhD, Amsterdam UMC, Academic Medical Center, Department of Radiology and Nuclear Medicine, G1-222 Meibergdreef 91105 AZ Amsterdam, the Netherlands; e-mail: l.reneman@amsterdamumc.nl

\footnotetext{
-- Indicates open access to non-subscribers at www.ajnr.org

Indicates article with supplemental on-line appendix, photo, and trial protocol.

Indicates article with supplemental on-line photo.

http://dx.doi.org/10.3174/ajnr.A6560
} 


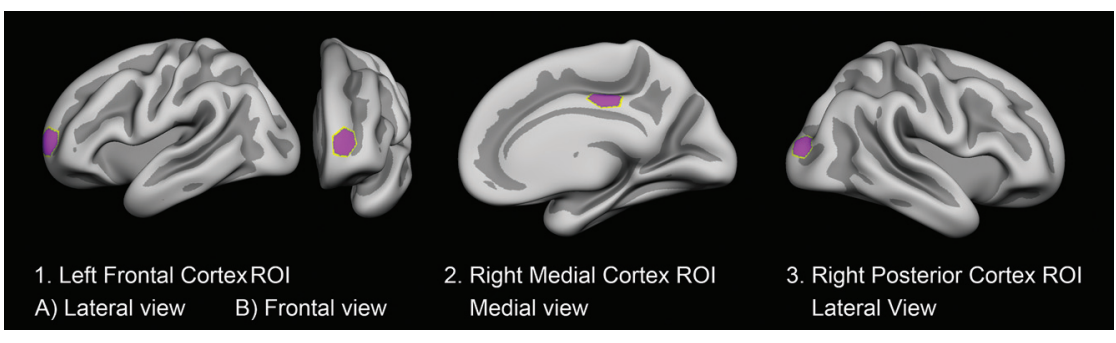

FIG 1. ROIs investigated. Cortical ROls were determined on the basis of the MNI coordinates of the vertices corresponding to peak group differences in the observational prospective study of psychostimulant treatment and the developing cortex by Shaw et al. ${ }^{9}$ Labels were created at the corresponding vertices on the FreeSurfer average surface. The labels were dilated 15 times, resulting in hexagon-shaped ROIs, covering approximately $550 \mathrm{~mm}^{2}$ when transformed to the individual participants' brain surfaces. Shown here are the following: 1) the left frontal cortex ROI in lateral $(A)$ and frontal $(B)$ view; 2 ) the right medial cortex ROI in the medial view; and 3) the right posterior cortex $\mathrm{ROI}$ in the lateral view.

some evidence for medication-associated differences for cortical thickness changes, ${ }^{9}$ but an absence of such evidence for basal ganglia trajectories. ${ }^{5}$ However, such observational studies are limited by possible selection effects. Moreover, the cortical differences observed have been in terms of greater thinning of the cortex in the right medial frontal motor region, the left middle/inferior frontal gyrus, and the right posterior parieto-occipital region in children with ADHD not taking psychostimulants, ${ }^{9}$ and different effects of psychostimulant medication can be expected during development, when the brain changes at a higher rate than in adulthood. ${ }^{10}$

We recently reported age-dependent effects of methylphenidate on changes in cerebral blood flow of the dopaminergic system (our predefined primary aim), as measured by pharmacologic MR imaging, in young-versus-adult patients with ADHD. ${ }^{11}$ Because our RCT is the first study that examines the effects of methylphenidate on brain development, this means that there are only limited and indirect data available to perform a sample-size calculation. Our goal for this research was to be able to detect differences in the age-dependent effect of methylphenidate on the brain if these differences are in the magnitude of a standardized effect size of 1.25 (see also On-line Research Protocol, page 18). A secondary aim of the Effects of Psychotropic Drugs on Developing Brain (ePOD) study is to assess the effects of methylphenidate treatment on cortical changes in children and adults with ADHD in a double-blind RCT. Due to ongoing development of the dopaminergic system during childhood and adolescence, we hypothesized that methylphenidate treatment would be associated with reduced cortical thinning (rate of change) in the left frontal, right posterior, and right medial cortices ${ }^{9}$ and that this would be observed in children only, not in adults. In addition to testing this hypothesis, we also explored possible cortical effects in a vertex-wise whole-brain analysis and possible relations between cortical change and change in ADHD symptom severity.

\section{MATERIALS AND METHODS \\ Trial Design}

The ePOD-Methylphenidate RCT was a 16-week double-blind, randomized, placebo-controlled, multicenter trial of the use of methylphenidate and a blinded end point evaluation in psychostimulant treatment-naïve patients with ADHD. ${ }^{11,12}$ The length of the trial was dictated by the waiting list for treatment. In the Netherlands at the time of the execution of this trial, a waiting list of 17 weeks was very common before (child and adolescent) psychiatric evaluation could take place and treatment could commence. It is in this timeframe that the current study was executed, to ensure only a very small or no delay in the placebo group before active treatment could take place. The timeframe was thus chosen so that patients treated with a placebo would not be harmed by study participation (for further details, see the On-line Research Protocol). The primary outcome measure of the ePOD-Methylphendiate trial was to report on the effect modification by age of the methylphenidate treatment on the outgrowth of the dopaminergic system. ${ }^{11}$

Here, we report our secondary outcome. A prior article dealt with the effect of methylphenidate on white matter development, ${ }^{13}$ whereas this article assesses the effects on the rate of change in cortical thickness of selected ROIs assessed using structural MR imaging in exactly the same sample of children and adults with ADHD, randomly assigned to either placebo or active treatment with methylphenidate, pre- and posttreatment. The selection of ROIs was based on prior results from an observational prospective study of psychostimulant treatment and the developing cortex by Shaw et al. ${ }^{9}$ The ROIs chosen for investigation are shown in Fig 1. The study protocol applied the code of medical ethics and was registered by the Central Committee on Research Involving Human Subjects (an independent registry) on March 24, 2011 (identifier NL34509.000.10) and subsequently at the Netherlands National Trial Register (identifier NTR3103), with enrollment of the first patient on October 13, 2011. In addition, the institutional review board of the Academic Medical Center approved the study. The full protocol is described elsewhere. ${ }^{12}$ The trial ended on June 15, 2015, and was monitored by the Clinical Research Unit of the Academic Medical Center, University of Amsterdam, the Netherlands. All patients and parents or legal representatives of the children provided written informed consent.

\section{Participants}

Participants were psychostimulant treatment-naïve, 50 boys (1012 years of age) and 49 men (23-40 years of age), diagnosed as having ADHD and recruited through clinical programs at the Department of Child and Adolescent Psychiatry at Triversum (Alkmaar, the Netherlands), De Bascule Academic Center for Child and Adolescent Psychiatry (Amsterdam), and PsyQ mental health facility (The Hague). As laid out in the protocol, the cutoff point of 10-12 years of age for the pediatric population was chosen because the peak prevalence of ADHD has been found to be 
10 years of age, ${ }^{14}$ and also because several MR imaging parameters show greater age differences in early than later childhood. ${ }^{15}$ One hundred thirty-one individuals were screened for eligibility. All included met strict criteria for ADHD (all subtypes) according to the Diagnostic and Statistical Manual of Mental Disorders, 4th Edition $(D S M-I V)^{16}$ and were diagnosed by an experienced psychiatrist. Diagnoses were confirmed with diagnostic interviews. $^{17,18}$ More detailed inclusion and exclusion criteria are available in the On-line Appendix and On-line Research Protocol (see also Schrantee et al). ${ }^{11}$

\section{Intervention, Randomization, and Blinding}

Patients were randomly assigned to either methylphenidate or placebo treatment. ${ }^{11}$ The treating physicians prescribed the study medication under double-blind conditions on clinical guidance in accord with Dutch treatment guidelines. Medication dosages were titrated per individual, under supervision of a child and adolescent psychiatrist. Adult participants received coaching sessions, and parents of children received psychoeducation. Adherence to the study medication was monitored, and the compliance rate (0.0-1.0) was calculated (On-line Appendix).

\section{Outcomes}

Primary Outcome Measure of ROI Cortical Thickness Change. Imaging was performed using 1 of two 3T MR imaging systems (scanner 1, Intera; scanner 2, Achieva; Philips Healthcare). All participants were scanned on the same scanner pre- and postintervention. Anatomic 3D T1-weighted fast-field echo sequences were acquired and used for cortical surface analyses. Cortical thickness measures were derived using FreeSurfer (http://surfer. nmr.mgh.harvard.edu), Version 5.3. Cortical thickness was estimated vertex-wise across the brain surface using an automated approach. ${ }^{19-23}$ Scans were rated for the presence of motion and were excluded unless rated $(1=$ no sign of motion, or $2=$ minor signs of motion, but no major distortion and acceptable reconstruction). An ROI approach was considered the viable option, given the necessarily restricted participant numbers of this RCT. Montreal Neurological Institute (MNI) coordinates of the vertices corresponding to peak group differences in the observational study ${ }^{9}$ were converted to Talairach coordinates, and labels were created at the corresponding vertices on the FreeSurfer average surface. The MNI coordinates for peak group differences forming the basis for the cortical ROIs were as follows: left frontal: -26 , 61, 6; right medial frontal: 6, -23, 40; right posterior: 30, -93, 12. The labels were dilated 15 times, resulting in hexagon-shaped ROIs covering approximately $550 \mathrm{~mm}^{2}$ when transformed to the individual participant's brain surface (Fig 1). Surface measures were extracted from the individual participants (Fig 1 and Online Appendix).

\section{Statistical Analyses}

Analyses of ROI values were performed using SPSS, Version 24 (IBM), per reviewer request; analyses on handedness were later added, performed using SPSS, Version 26 (https://www.ibm.com/ analytics/data-science/predictive-analytics/spss-statistical-software). One-way ANOVA was used to test differences in baseline brain characteristics, scan interval, scan movement ratings, compliance rates, and age for children and adults separately. Linear mixed models with age group (children, adults) $\times$ treatment group (placebo, methylphenidate) $\times$ time (pre-, posttreatment), as well as the main effects and interactions of each, were set up to test interaction effects on rates of change, using maximum likelihood estimation, with the covariance structure set to compound symmetry. For further analysis of medication effects per age group, we split the sample by age and set up similar linear mixed models with treatment group (placebo, methylphenidate) $\times$ time (pre-, posttreatment), as well as the main effects and interactions of each, to test interaction effects on rates of change within each age group for all ROIs separately. The significance level was set at $P<.05$.

While the ROI analysis was the primary outcome measure given the small number, an exploratory vertex-wise analysis on the whole cortical mantle ${ }^{24}$ was also performed (see On-line Appendix for details). In addition, cortical ROI change scores were correlated with the change in symptom severity preand posttreatment to explore possible relations.

\section{RESULTS}

\section{Randomization and Baseline Characteristics}

A total of 99 patients with ADHD were randomized to methylphenidate or placebo treatment. After randomization, 1 adult disclosed previous methylphenidate treatment and was therefore excluded from the analyses. On inspection of the scans, it was found in the child placebo group that one subject had a relatively large posterior cyst causing possible displacement of brain ROIs and another had an abnormality in the right frontal lobe that seemed to affect reconstructions of surfaces in the lateral cortex (by neuroradiologic follow-up thought to possibly be a benign tumor). Both of these individuals were therefore excluded from the current analyses. Forty-eight children and 47 adults were included in the primary analysis (CONSORT flow diagram, based on a template from http://www.consort-statement.org/consortstatement/flow-diagram, Fig 2). Of these, 1 child and 2 adults had invalid baseline data, and 1 child had invalid follow-up data due to scan motion or poor scan quality, leading to flawed image reconstruction. Note that 1 of the adults whose baseline data did not pass quality control was also among the ones who discontinued intervention. Treatment medication adherence data were missing for 1 child in the placebo group and for 3 children in the methylphenidate treatment group. No serious adverse events were noted in any of the individuals studied.

Sample characteristics are given in the Table (for in-depth clinical descriptives and behavioral measures used, see the On-line Appendix and Schrantee et $\mathrm{al}^{11}{ }^{11}$ Schmand et $\mathrm{al}^{25}$ Rosler et $\mathrm{al}^{26}{ }^{26}$ Oosterlaan et $\mathrm{al}^{27}$ and Wechsler ${ }^{28}$ ). There were no significant differences in baseline age, ADHD symptom severity, reported handedness (proportion right-handed), estimated intelligence quotient, or ROI brain characteristics or scan interval or movement ratings between the placebo and medication groups. For the children, no tendency $(P<.10)$ toward differences between treatment groups was observed. However, for the adults, there was a tendency toward a thicker cortex in the right posterior ROI $(P=.072)$ at baseline in the placebo group and a greater compliance rate in the treatment group $(P=.096)$. Because outcome ROI analyses were 


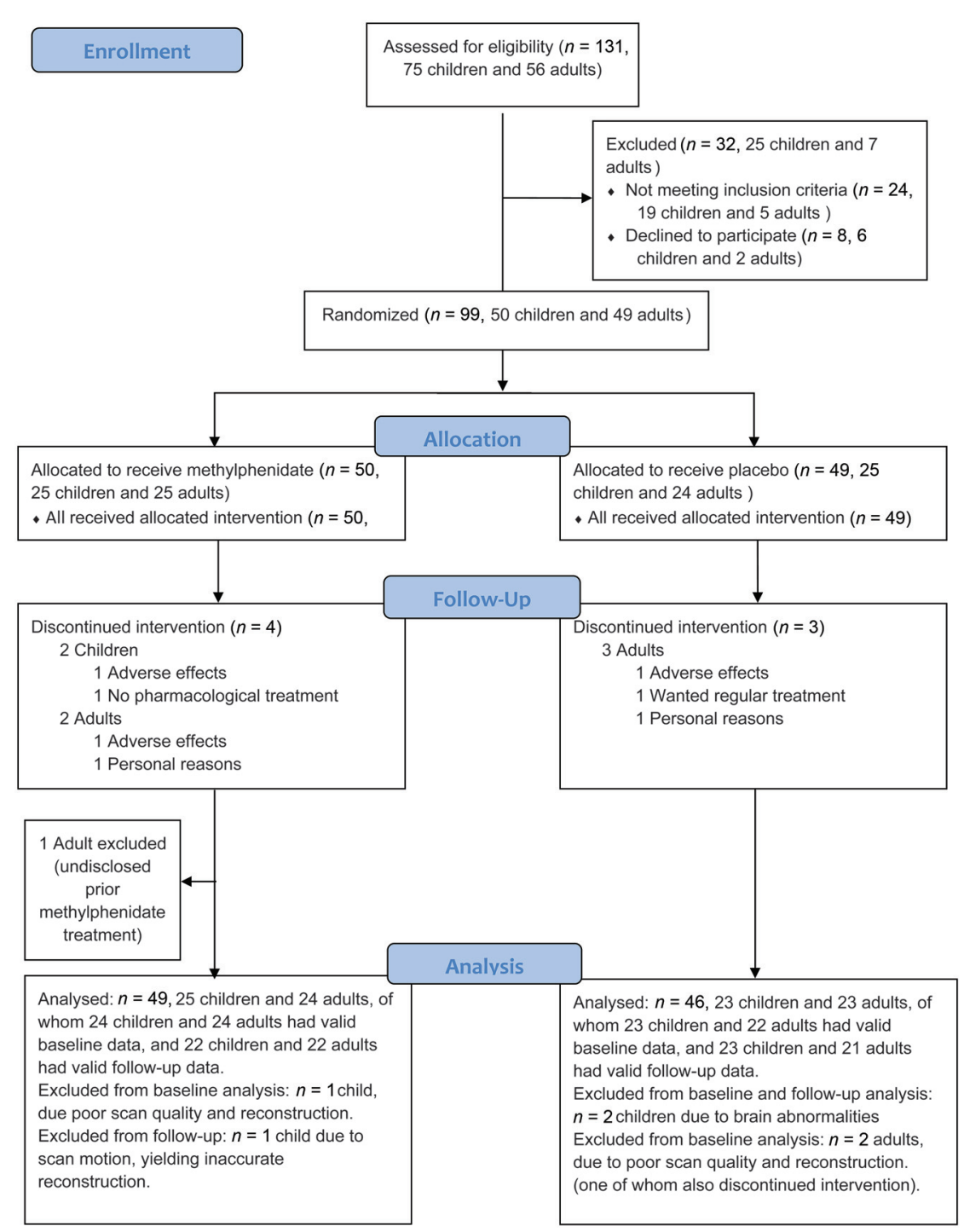

FIG 2. Consolidated standards of reporting trials flow diagram. Patients were randomized to either methylphenidate or placebo.

for longitudinal cortical thickness change only, we chose not to correct for intracranial volume in the analyses.

\section{Main Outcome of the ROI Cortical Thickness Change}

Cortical thickness values for the groups pre- and posttreatment are shown in Fig 3, and individual data are shown in On-line Fig 1. Analyses using linear mixed models with scan time as a repeat measure $\times$ age $\times$ medication group showed, for the right medial cortex ROI a main effect of age $(F[1,95.606]=199.713, P<.001)$, likely reflecting expected age-related thinning, and an interaction of medication group $\times$ age group $\times$ time $(F[1,88.825]=4.316$, $P=.041)$. As shown in Fig 3 , in the children, the methylphenidate group showed increasing thickness, while the placebo group showed cortical thinning, whereas in adults, both groups showed cortical thinning. Although there were no significant baseline differences, slight differences were seen at baseline throughout the ROIs across age and medication groups, which also contributed to the identified interaction. The individual data plots did, however, not reveal striking outliers at any time point. Relatively lesser thinning in children in the methylphenidate treatment group was also seen in the right posterior cortex ROI, but not in the left frontal cortex ROI. However, no significant 3-way interaction effects of medication group $\times$ age group $\times$ time were found, except for the right medial cortex ROI. Other significant fixed effects in these analyses were limited to a main effect of age on the left frontal cortex $(F$ $[1,96.308]=46.788, P<.001)$ and the right posterior cortex $(F[1,96.289]=$ 51.361, $P<.001$ ), for which an interaction of age group $\times$ medication group was also found $(F[1,96.289]=3.973$, $P=.049$ ). This effect appeared to be due to the child medication group having, on average, thicker cortices with the adult medication group having, on average, thinner cortices than the same-age placebo groups.

The analyses per age group confirmed an interaction of medication group $x$ time $(F[1,44.893]=4.793, P=.034)$ in the young group only for the right medial cortex ROI. In the per-age group analyses, no other significant interaction effects were found, only a main effect of the medication group in the adults for the right posterior cortex ROI $(F[1,47.274]=4.483, P=.040)$, reflecting a thicker cortex in the adult placebo group at both time points. However, there were no trends toward the interaction of medication group $x$ time for this ROI $(P=.986)$. For comparison with previous results from the longer observational study from which the ROIs were derived, ${ }^{9}$ the present change rate for the right medial cortex ROI was converted to a rate of change per year, shown in Fig 4. The mean rates of change in cortical thickness for this region were quite similar across studies, but confidence intervals were larger in the present study.

The exploratory vertex-wise whole-brain analyses did not reveal any significant interaction between group and treatment on the thickness rate of change in any region when corrected for multiple comparisons (false discovery rate, $P<.05$ ). One-way ANOVA confirmed an effect of group on symptom change in the present sample, with post-, minus pre-treatment scores being lower for the methylphenidate than for the placebo groups $(F[1,42]=5.551, P=.023$, and $F[1,41]=5.857, P=.020$ for children and adults, respectively). Mean posttreatment ADHD symptom-severity scores in children were $30.2 \pm 10.1(n=22)$ versus $21.7 \pm 10.5(n=22)$ for the placebo and methylphenidate groups, 
Sample characteristics for those included at baseline with valid MRIs, age, intelligence quotient, reported handedness, ADHD symptom severity, and brain ROls, as well as scan movement ratings at baseline and follow-up and scan interval and compliance rates for those included in the analyses, divided by age and medication group ${ }^{a}$

\begin{tabular}{|c|c|c|c|c|c|c|c|c|}
\hline & \multicolumn{4}{|c|}{ Children } & \multicolumn{4}{|c|}{ Adults } \\
\hline & \multicolumn{2}{|c|}{$\begin{array}{c}\text { Methylphenidate } \\
\text { Hydrochloride } \\
(n=25)\end{array}$} & \multicolumn{2}{|c|}{ Placebo $(n=23)$} & \multicolumn{2}{|c|}{$\begin{array}{c}\text { Methylphenidate } \\
\text { Hydrochloride } \\
(n=24)\end{array}$} & \multicolumn{2}{|c|}{ Placebo $(n=23)$} \\
\hline & Mean & SD & Mean & SD & Mean & SD & Mean & SD \\
\hline Age at baseline (yr) & 11.4 & 0.8 & 11.4 & 0.9 & 28.2 & 4.4 & 29.0 & 5.0 \\
\hline Estimated intelligence quotient ${ }^{b}$ & 104.8 & 21.0 & 102.8 & 15.7 & 107.9 & 8.8 & 107.9 & 6.4 \\
\hline ADHD symptom severity & 36.6 & 6.2 & 38.4 & 8.2 & 31.8 & 9.9 & 31.0 & 9.9 \\
\hline Proportion right-handed & 0.840 & 0.374 & 0.913 & 0.288 & 0.958 & 0.204 & 0.826 & 0.388 \\
\hline $\begin{array}{l}\text { Left frontal cortex thickness, } \\
\qquad \mathrm{Tpl}(\mathrm{mm})^{c}\end{array}$ & 2.805 & 0.229 & 2.763 & 0.236 & 2.471 & 0.170 & 2.553 & 0.164 \\
\hline $\begin{array}{l}\text { Right medial cortex thickness, } \\
\text { Tpl }(\mathrm{mm})^{c}\end{array}$ & 2.724 & 0.115 & 2.770 & 0.097 & 2.434 & 0.128 & 2.438 & 0.120 \\
\hline $\begin{array}{l}\text { Right posterior cortex } \\
\text { thickness, Tpl }(\mathrm{mm})^{c}\end{array}$ & 2.409 & 0.142 & 2.384 & 0.172 & 2.151 & 0.157 & 2.223 & 0.096 \\
\hline Scan movement $\mathrm{Tpl}^{\mathrm{c}}$ & 1.2 & 0.4 & 1.2 & 0.4 & 1.0 & 0.0 & 1.1 & 0.2 \\
\hline Scan movement Tp2 ${ }^{d}$ & 1.3 & 0.5 & 1.2 & 0.4 & 1.1 & 0.2 & 1.0 & 0.0 \\
\hline Compliance rate percentage $\mathrm{e}^{\mathrm{e}}$ & 83.5 & 15.5 & 79.0 & 18.1 & 90.0 & 8.0 & 85.7 & 8.4 \\
\hline Scan interval (day) & 122.3 & 4.2 & 121.4 & 3.9 & 110.8 & 27.5 & 111.7 & 24.6 \\
\hline
\end{tabular}

Note:-Tp indicates time point; Tp1, baseline time point; and Tp2, follow-up time point.

${ }^{a}$ There were no significant $(P<.05)$ differences in these characteristics across participants randomized to methylphenidate or placebo treatment.

${ }^{\mathrm{b}}$ Available for 21 children and 22 adults in the placebo group and all 25 children and 22 adults in the methylphenidate group.

cAvailable for those with scans included at baseline: 23 children and 22 adults in the placebo group and 24 children and 24 adults in methylphenidate group.

${ }^{d}$ Available for those with scans included at follow-up: 23 children and 21 adults in placebo group and 22 children and 22 adults in methylphenidate group.

e Available for 22 children and 21 adults in the placebo group and 20 children and 22 adults in the methylphenidate group.

${ }^{f}$ Available for those included who did not drop out: 23 children and 21 adults in placebo group and 23 children and 22 adults in methylphenidate group.

respectively. Mean posttreatment $\mathrm{ADHD}$ symptom-severity scores in adults were $26.5 \pm 7.9(n=21)$ versus $20.1 \pm 6.7(n=22)$ for the placebo and methylphenidate groups, respectively. However, there were no significant relationships between symptom-severity change and the estimated rate of ROI cortical change per year in either children or adults.

\section{DISCUSSION}

The present results yield partial support for the hypothesis that methylphenidate treatment in development is associated with reduced cortical thinning, ${ }^{9}$ as identified here in the right medial cortex. The 3-way interaction of treatment group $\times$ age group $\times$ time for this region also indicates that similar effects were not seen in adulthood, in line with the hypothesis. However, for the 2 other cortical ROIs previously indicated, ${ }^{9}$ in the left frontal and right posterior cortices, no significant medication group $\times$ time interactions were observed in the current RCT.

The only partial overlap and noted discrepancies of findings across the current study and the previous observational study of children and adolescents ${ }^{9}$ may be due to several factors: First, in the observational study, decisions regarding psychostimulant treatment were the joint responsibility of the patients, their families, and their physicians, ${ }^{9}$ whereas in this study, participants were randomized. While there were no significant differences in baseline characteristics in terms of age, ADHD symptom severity, and clinical impairment across treatment groups in either study, ${ }^{9,11}$ it may still be more likely that groups self-selecting medication/no medication differed on some dimensions. Second, the current follow-up interval is considerably shorter than that of the observational study, ie, 4 months versus 4 years. There are substantial cortical changes in the relevant age ranges, ${ }^{10,29-32}$ and it may be that additional or other effects would be seen in a longer RCT. However, for ethical and practical reasons, a much longer RCT with patients with ADHD, children in particular, is deemed impossible to perform. Of note, when converted to the mean rate of change per year, the present results for the right medial cortex resemble those found across longer time intervals in the observational study. ${ }^{9}$ Third, the MR imaging measures used in the previous observational study ${ }^{9}$ and the present one vary in terms of scanner platforms and field strengths (1.5T versus $3 \mathrm{~T}$ ), sequence parameters, and cortical reconstructions and segmentations. The observational study ${ }^{9}$ used CIVET (https:// sourceforge.net/projects/civet/) software for cortical reconstruction and thickness estimation, whereas FreeSurfer ${ }^{19-23}$ was used in the present study. The measurement of cortical thickness requires that the analysis be performed at the nodes of a $3 \mathrm{D}$ polygonal mesh, ${ }^{33}$ capturing the distance between the WM surface and the intersection between the outer cortical boundary and the CSF. This is in accordance with a geometric definition, which can vary across methods, so the way cortical thickness is quantified is of importance for the end results. Of note, the normal maturational cortical trajectories measured by CIVET and FreeSurfer can be discrepant. ${ }^{34}$ Moreover, the exact re-creation of the cortical effect sites identified in the observational study ${ }^{9}$ was not possible; therefore, smaller ROIs were drawn around the peak effects.

Methodologic issues aside, the present results yield an indication toward thicker regional cortices with methylphenidate treatment in childhood specifically, as shown in the 3-way interaction of treatment group $\times$ age group $\times$ time for the right medial cortex, with absence of any treatment group $\times$ time interactions in 
Children
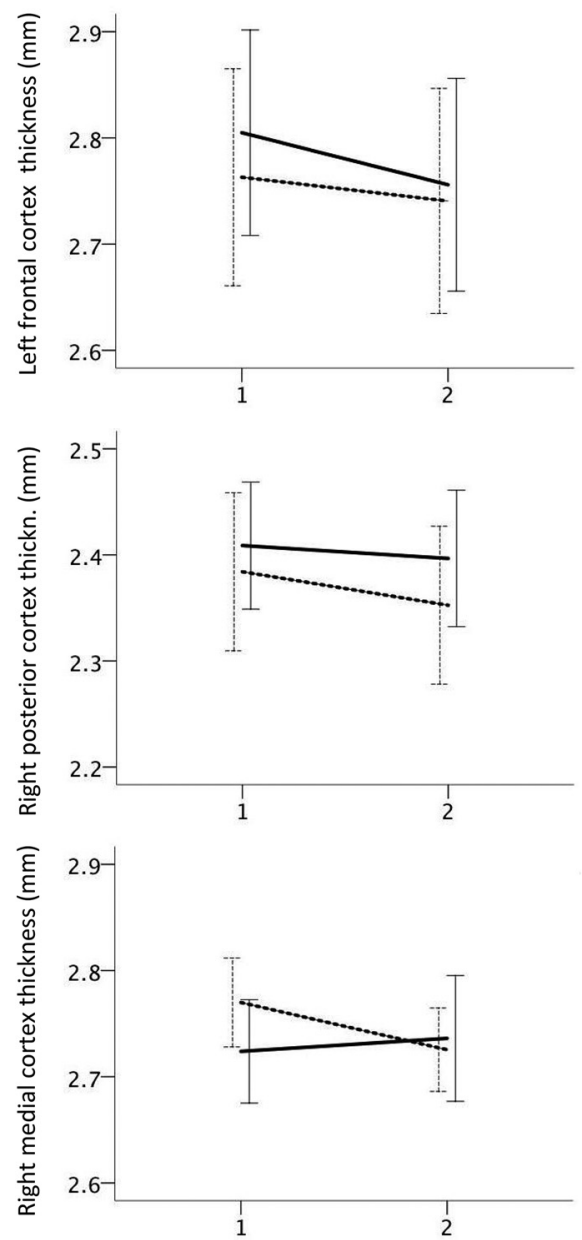

Adults
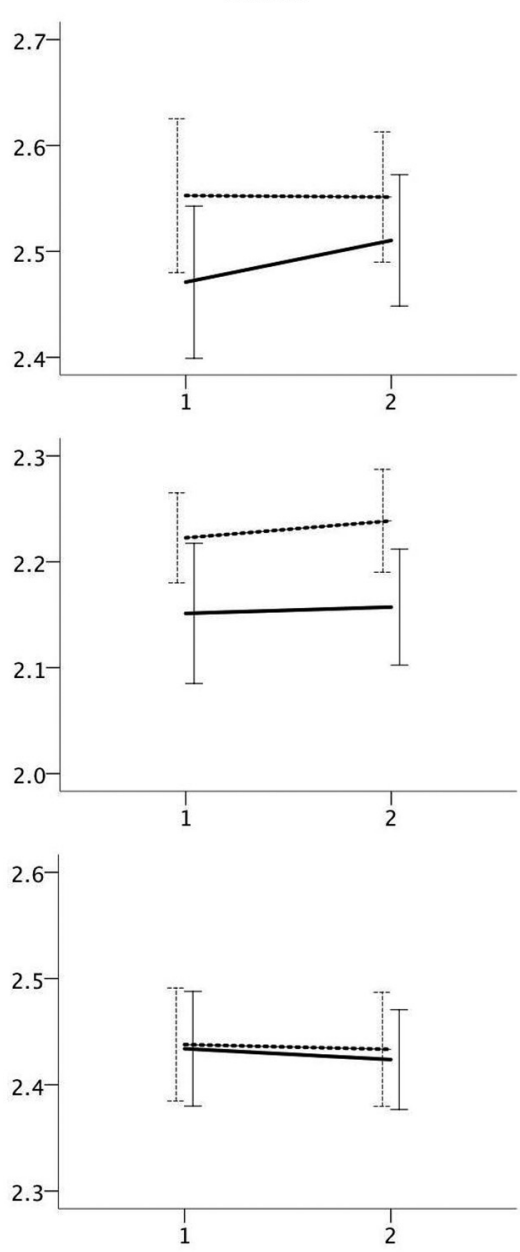

epresent $95 \%$ C

FIG 3. Differences in rates of cortical thickness change of the ROIs in children and adults taking methylphenidate or placebo medication. For each ROI, at the $y$-axis, cortical thickness in millimeters (in left frontal, right posterior, and right medial cortices) is shown at baseline ( $x$-axis time point 1 ) and at the end point ( $x$-axis time point 2 ) of the trial. Note that as cortical thickness differs across regions and age groups, different origin values for cortical thickness are given across panels to enable visualization, but the scale is otherwise the same, and increments on the y-axes invariably represent $0.1 \mathrm{~mm}$. Mean rates of change are shown with dotted lines for the placebo group and solid lines for the methylphenidate group. Error bars represent the $95 \%$ confidence intervals. adults. This finding corresponds to evidence from animal models, indicating that psychotropic drugs affect the brain in a differential manner depending on the age of exposure. ${ }^{35,36}$ The results also align with findings of agedependent effects of methylphenidate on cerebral perfusion and the blood flow response of the dopaminergic system in the same sample studied here. ${ }^{37}$ Hence, the present data on cortical thickness changes are in line with the neurochemical imprinting hypothesis, ${ }^{36}$ to some extent also being at play at a macrostructural level.

To the extent that brain differences among ADHD case-controls are predominantly found in children rather than adults, as was recently reported, ${ }^{7,8}$ it is also given that such differences can only be influenced when they are present in childhood. In large recent crosssectional studies on observational cohorts, no effect of psychostimulant medication on subcortical volumes ${ }^{8}$ or the cortical thickness of regions showing patient-control group differences (fusiform gyrus, temporal pole) ${ }^{7}$ were found. There are, as mentioned above, multiple methodologic challenges to studying the effects of medication in a pure observational setting. The absence of such observational cross-sectional medication effects on cortical thickness could mean that such effects are not present in the studied regions or that any such effects are transient. It could, however, also be that medication may cause persistent changes, even if they can only be detected at the level of brain structure for a limited time.
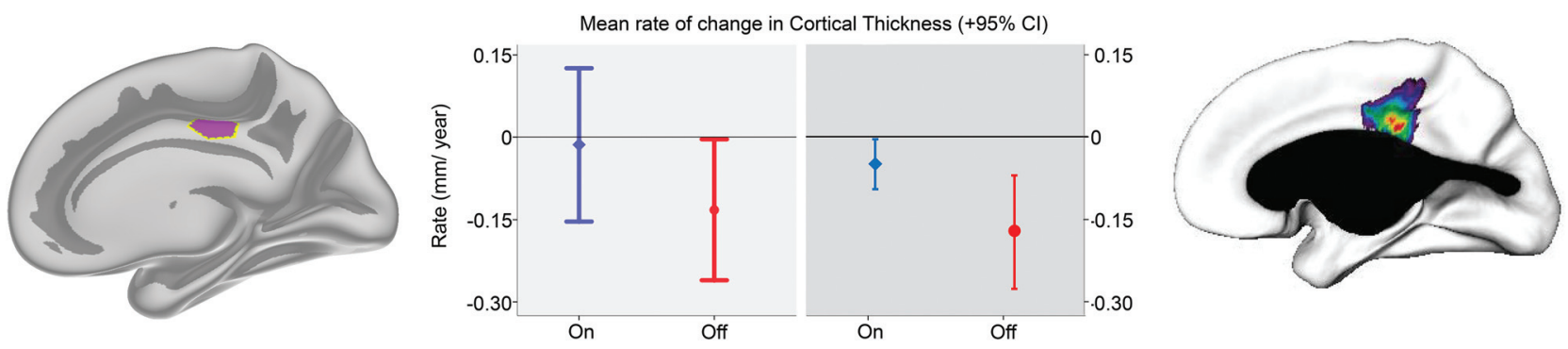

FIG 4. Mean rate of change in cortical thickness estimated per year for the present RCT and the previous observational study for the right medial cortex region. For children receiving methylphenidate (blue) and placebo (red), cortical change values for the right medial ROI across the duration of the present RCT were converted to an estimated mean rate of change in cortical thickness per year (left panel). This was done for comparison with previous results (right panel) from the observational study from which the ROI was derived (Shaw et al ${ }^{9}$ ), reporting on the mean rate of cortical change per year across a period of about 4 years. 


\section{Limitations}

There are several limitations to the present study. First, the sample was too small for detection of effects in a whole-brain analysis. Thus, although there were no significant baseline differences, slight differences were seen throughout the ROIs across age and medication groups, and baseline differences also contributed to the identified interaction. The vast normal variation in brain anatomy, including cortical measures, ${ }^{38-40}$ unfortunately makes it unlikely that relatively small groups will have completely identical values. Second, because we did not detect significant relationships between changes in cortical ROIs and changes in symptom severity, the functional significance remains uncertain. ADHD symptom severity was not related to subcortical differences in a recent cross-sectional mega-analysis either, ${ }^{8}$ so direct morphometricfunctional relationships may not necessarily be expected. However, it would be of interest in future larger studies to investigate whether both baseline and longitudinal differences in cortical thickness are associated with a differential response to medication. Third, the follow-up interval was relatively short, and while changes in cortical thickness can be reliably detected across time at intervals even shorter than the present ones, ${ }^{41}$ medication effects may develop differently across a longer time. It is unknown why the right medial frontal cortical region, rather than the left frontal and left posterior cortical ROIs, showed the hypothesized effect in the present study. There was no reason to expect a differential strength of effects based on the previous observational study. ${ }^{9}$ We do not believe it is necessary to speculate about possible causes for this in this relatively small sample in which we also do not find correlations with symptom severity in any of the ROIs. Furthermore, it is beyond the scope of the present article to address functional imaging changes, but changes in cortical thickness with medication may likely also relate to and possibly interact with functional imaging changes, such as blood oxygen level-dependent functional signal variability. ${ }^{42}$

Finally, macrostructural MR imaging measures cannot pinpoint the neural changes underlying the observed differences in thickness. Neurogenesis does not take place in the cortex in these age ranges, ${ }^{43}$ but a number of other processes may be at play, inclusive of differences in growth and regressive changes, astrocytes, dendritic branching, synaptic pruning, intracortical myelination, dendritic spine formation, and vascular changes. ${ }^{44-50}$ Preferably, animal models should combine high-resolution MR imaging and histologic methods to elucidate the neural fundament. The present results warrant replication in larger groups and possibly with longer followup, to determine whether the effects are permanent and whether they have long-term functional correlates.

\section{CONCLUSIONS}

The results of this RCT yield partial support for the hypothesis that methylphenidate treatment in development is associated with reduced cortical thinning in childhood, but not adulthood. This was identified here in the right medial cortex, but not in 2 other cortical ROIs previously indicated. ${ }^{9}$ Because significant relationships between changes in cortical ROIs and changes in symptom severity were not observed, the functional significance remains uncertain. Replication of the effects in prolonged studies in larger groups would be required to determine both possible temporal dynamics and functional significance of cortical effects.

Disclosures: Inge Amlien-RELATED: Grant: UNINETT Sigma2 NS9084S, Comments: computer resources (storage/processing). Atle BjørnerudUNRELATED: Employment: Oslo University Hospital and University of Oslo.

\section{REFERENCES}

1. Burcu M, Zito JM, Metcalfe L, et al. Trends in stimulant medication use in commercially insured youths and adults, 2010-2014. JAMA Psychiatry 2016;73:992-93 CrossRef Medline

2. Trip AM, Visser ST, Kalverdijk LJ, et al. Large increase of the use of psycho-stimulants among youth in the Netherlands between 1996 and 2006. Br J Clin Pharmacol 2009;67:466-68 CrossRef Medline

3. Group M. National Institute of Mental Health Multimodal Treatment Study of ADHD follow-up: 24-month outcomes of treatment strategies for attention-deficit/hyperactivity disorder. Pediatrics 2004;113:754-61 CrossRef Medline

4. Swanson J, Greenhill L, Wigal T, et al. Stimulant-related reductions of growth rates in the PATS. J Am Acad Child Adolesc Psychiatry 2006;45:1304-13 CrossRef

5. Shaw P, De Rossi P, Watson B, et al. Mapping the development of the basal ganglia in children with attention-deficit/hyperactivity disorder. J Am Acad Child Adolesc Psychiatry.2014;53:780-89.e711 CrossRef Medline

6. Shaw P, Malek M, Watson B, et al. Development of cortical surface area and gyrification in attention-deficit/hyperactivity disorder. Biol Psychiatry 2012;72:191-97 CrossRef Medline

7. Hoogman M, Muetzel R, Guimaraes JP, et al. Brain imaging of the cortex in ADHD: a coordinated analysis of large-scale clinical and population-based samples. Am J Psychiatry 2019;176:531-42 CrossRef Medline

8. Hoogman M, Bralten J, Hibar DP, et al. Subcortical brain volume differences in participants with attention-deficit hyperactivity disorder in children and adults: a cross-sectional mega-analysis. Lancet Psychiatry 2017;4:310-19 CrossRef Medline

9. Shaw P, Sharp WS, Morrison M, et al. Psychostimulant treatment and the developing cortex in attention-deficit hyperactivity disorder. Am J Psychiatry 2009;166:58-63 CrossRef Medline

10. Tamnes CK, Walhovd KB, Dale AM, et al; Alzheimer's Disease Neuroimaging Initiative. Brain development and aging: overlapping and unique patterns of change. Neuroimage 2013;68:63-74 CrossRef Medline

11. Schrantee A, Tamminga HG, Bouziane C, et al. Age-dependent effects of methylphenidate on the human dopaminergic system in young vs adult patients with attention-deficit/hyperactivity disorder: a randomized clinical trial. JAMA Psychiatry 2016;73:955-62 CrossRef Medline

12. Bottelier MA, Schouw ML, Klomp A, et al. The effects of Psychotropic drugs On Developing brain (ePOD) study: methods and design. BMC Psychiatry 2014;14:48 CrossRef Medline

13. Bouziane C, Filatova OG, Schrantee A, et al. White matter by diffusion MRI following methylphenidate treatment: a randomized control trial in males with attention-deficit/hyperactivity disorder. Radiology 2019;293:186-92 CrossRef Medline

14. Burd L, Klug MG, Coumbe MJ, et al. Children and adolescents with attention-deficit-hyperactivity disorder. 1: prevalence and cost of care. J Child Neurol 2003;18:555-61 CrossRef Medline

15. Ben Bashat D, Ben Sira L, Graif M, et al. Normal white matter development from infancy to adulthood: comparing diffusion tensor and high b value diffusion weighted MR images. J Magn Reson Imaging 2005;21:503-11 CrossRef Medline

16. Association AP. Diagnostic and Statistical Manual of Mental Health Disorders. 4th ed. American Psychiatric Association; 1994

17. Ferdinand R, van der Ende J, Nimh DI. Diagnostic Interview Schedule for Children (in Dutch). Erasmus MC-Sophia; 2000 
18. Kooij J, Francken M. Diagnostic Interview for ADHD in Adults (DIVA). 2010. https://www.divacenter.eu/Content/Downloads/DIVA_ 2_NL_FORMULIER.pdf. Accessed April 28, 2020

19. Fischl B, Sereno MI, Dale AM. Cortical surface-based analysis. II: inflation, flattening, and a surface-based coordinate system. Neuroimage 1999;9:195-207 CrossRef Medline

20. Fischl B, Dale AM. Measuring the thickness of the human cerebral cortex from magnetic resonance images. Proc Natl Acad Sci U S A 2000;97:11050-55 CrossRef Medline

21. Dale AM, Sereno MI. Improved localization of cortical activity by combining EEG and MEG with MRI cortical surface reconstruction: a linear approach. J Cogn Neurosci 1993;5:162-76 CrossRef Medline

22. Dale AM, Fischl B, Sereno MI. Cortical surface-based analysis, I: segmentation and surface reconstruction. Neuroimage 1999;9:17994 CrossRef Medline

23. Segonne F, Grimson E, Fischl B. A genetic algorithm for the topology correction of cortical surfaces. Inf Process Med Imaging 2005;19:393-405 Medline

24. Reuter M, Schmansky NJ, Rosas HD, et al. Within-subject template estimation for unbiased longitudinal image analysis. Neuroimage 2012;61:1402-18 CrossRef Medline

25. Schmand B, Bakker D, Saan R, et al. The Dutch Reading Test for Adults: a measure of premorbid intelligence level (in Dutch). Tijdschr Gerontol Geriatr 1991;22:15-19 Medline

26. Rosler M, Retz W, Thome J, et al. Psychopathological rating scales for diagnostic use in adults with attention-deficit/hyperactivity disorder (ADHD). Eur Arch Psychiatry Clin Neurosci 2006;256 (Suppl 1):i3-11 CrossRef Medline

27. Oosterlaan J, Scheres A, Antrop I, et al. Vragenlijst voor Gedragsproblemen bij Kinderen (VvGK). [Dutch Translation of the Disruptive Behavior Disorders Rating Scale]. Harcourt Test Publishers; 1998

28. Wechsler D. Manual for the Wechsler Intelligence Scale for ChildrenRevised. Psychological Corporation; 1974

29. Walhovd KB, Tamnes CK, Bjornerud A, et al. Maturation of corticosubcortical structural networks: segregation and overlap of medial temporal and fronto-striatal systems in development. Cereb Cortex 2015;25:1835-41 CrossRef Medline

30. Tamnes $\mathrm{CK}$, Walhovd $\mathrm{KB}$, Grydeland $\mathrm{H}$, et al. Longitudinal working memory development is related to structural maturation of frontal and parietal cortices. J Cogn Neurosci 2013;25:1611-23 CrossRef Medline

31. Fjell AM, Grydeland H, Krogsrud SK, et al. Development and aging of cortical thickness correspond to genetic organization patterns. Proc Natl Acad Sci U S A 2015;112:15462-67 CrossRef Medline

32. Walhovd KB, Krogsrud SK, Amlien IK, et al. Neurodevelopmental origins of lifespan changes in brain and cognition. Proc Natl Acad Sci U S A 2016;113:9357-62 CrossRef Medline

33. Lerch JP, Evans AC. Cortical thickness analysis examined through power analysis and a population simulation. Neuroimage 2005;24:16373 CrossRef Medline

34. Walhovd KB, Fjell AM, Giedd J, et al. Through thick and thin: a need to reconcile contradictory results on trajectories in human cortical development. Cereb Cortex 2017;27:1472-81 CrossRef Medline

35. Urban KR, Waterhouse BD, Gao WJ. Distinct age-dependent effects of methylphenidate on developing and adult prefrontal neurons. Biol Psychiatry 2012;72:880-88 CrossRef Medline

36. Andersen SL, Navalta CP. Altering the course of neurodevelopment: a framework for understanding the enduring effects of psychotropic drugs. Int $J$ Dev Neurosci 2004;22:423-40 CrossRef Medline

37. Schrantee A, Mutsaerts $\mathrm{H}$, Bouziane $\mathrm{C}$, et al. The age-dependent effects of a single-dose methylphenidate challenge on cerebral perfusion in patients with attention-deficit/hyperactivity disorder. Neuroimage Clin 2017;13:123-29 CrossRef Medline

38. Tamnes CK, Ostby Y, Walhovd KB, et al. Neuroanatomical correlates of executive functions in children and adolescents: a mag netic resonance imaging (MRI) study of cortical thickness. Neuropsychologia 2010;48:2496-2508 CrossRef Medline

39. Tamnes CK, Ostby Y, Fjell AM, et al. Brain maturation in adolescence and young adulthood: regional age-related changes in cortical thickness and white matter volume and microstructure. Cereb Cortex 2010;20:534-48 CrossRef Medline

40. Storsve AB, Fjell AM, Tamnes CK, et al. Differential longitudinal changes in cortical thickness, surface area and volume across the adult life span: regions of accelerating and decelerating change. Neurosci 2014;34:8488-98 CrossRef Medline

41. Engvig A, Fjell AM, Westlye LT, et al. Effects of memory training on cortical thickness in the elderly. Neuroimage 2010;52:1667-76 CrossRef Medline

42. Pur DR, Eagleson RA, de Ribaupierre A, et al. Moderating effect of cortical thickness on BOLD signal variability age-related changes. Front Aging Neurosci 2019;11:46 CrossRef Medline

43. Bhardwaj RD, Curtis MA, Spalding KL, et al. Neocortical neurogenesis in humans is restricted to development. Proc Natl Acad Sci U S A 2006;103:12564-68 CrossRef Medline

44. Zatorre RJ, Fields RD, Johansen-Berg H. Plasticity in gray and white: neuroimaging changes in brain structure during learning. Nat Neurosci 2012;15:528-36 CrossRef Medline

45. Shafee R, Buckner RL, Fischl B. Gray matter myelination of 1555 human brains using partial volume corrected MRI images. Neuroimage 2015;105:473-85 CrossRef Medline

46. Huttenlocher PR, De Courten C, Garey LJ, et al. Synaptic development in human cerebral cortex. Int J Neurol 1982;16-17:144-54 Medline

47. Huttenlocher PR, Dabholkar AS. Regional differences in synaptogenesis in human cerebral cortex. The Journal of Comparative Neurology 1997;387:167-78 CrossRef Medline

48. Huttenlocher PR. Morphometric study of human cerebral cortex development. Neuropsychologia 1990;28:517-27 CrossRef Medline

49. Huttenlocher PR. Synapse elimination and plasticity in developing human cerebral cortex. Am J Ment Defic 1984;88:488-96 Medline

50. Huttenlocher PR. Synaptic density in human frontal cortex: developmental changes and effects of aging. Brain Res 1979;163:195-205 CrossRef Medline 